\title{
TEST RESULTS ON CAPACITOR COMMUTATION CHARGING TYPE OF RESONANT POWER SUPPLY FOR SYNCHROTRON RING MAGNETS
}

\author{
Dong Sai, Yao Chenggui, Zhang Yunxiang \\ National Synchrotron Radiation Laboratory, University of Science and Technology of China \\ Hefei, Anhui 230029, P.R.China, E-mail: sdong@ ustc.edu.cn
}

\begin{abstract}
A new dual resonant type current ramping power supply was built and tested. This paper describes experimental results on several different current waveforms: a triangular waveform with flat bottom, a trapezoidal wave with flat bottom and a waveform with reduced fall time. This paper also analyses the advantages of this type of power supply and the potential applications.
\end{abstract}

\section{INTRODUCTION}

Conventionally, synchrotron ring magnet power supplies adopt resonant system with DC-bias ${ }^{[1,2,3,4,]}$ or phase-controlled rectifiers ${ }^{[5,6,7]}$. The former, mostly used for fast-cycling synchrotrons, requires highly precise resonant frequency, and fixed cycle time. The advantage is that it eliminates the requirement of a large amount of reactive power. The latter approach, mostly used for slow-cycling synchrotrons, needs a large amount of reactive power compensation. But the load current waveforms and the cycle time can vary.

The dual resonant power supply ${ }^{[8,9,10]}$ can produce flat top and flat bottom current pulse. But most of research work is on theoretical analysis and experimental project. So far no practical application was reported. In 1996, M. R. Pavan Kumar \& J. M. S. Kim developed a circuit, namely, "HMSC" ${ }^{[11]}$. They consider that due to large reactive components used as energy storage device in the above-mentioned designs, these power supplies tend to have limited dynamic response. For the same reason, these designs also require high voltage power source.

In order to overcome these shortcomings, we propose a new type of resonant power supply. Its main advantages are:

the reactive power is eliminated; its reactive components reduced; and the circuit is simplified. The waveform of load current consists of rising and falling sections, a flat bottom and a flat top. The cycle time is adjustable, and it does not need high voltage power supplies. It is suitable for both fast and slow cycling synchrotrons.

Our research brings forth three main modifications:

- Insulated Grid Bipolar Transistors (IGBT) are used as the main switches,

- Electrolytic capacitors are used as energy store device.
- One DC regulator and one SCR are used to provide flat bottom current, and a large choke is eliminated.

Based on this idea, several different experiments of different load current waveforms with about $30 \mathrm{~A}$ peak flat top value have been conducted. The results are promising. We believe that this type of power supply design can be applied to future accelerator projects.

\section{PRINCIPLE AND WORKING PROCESS DESCRIPTION}

Figure 1 is a diagram of the main circuit. The parts framed in dotted lines are optional depending on whether non-zero flat bottom or flat top is required.

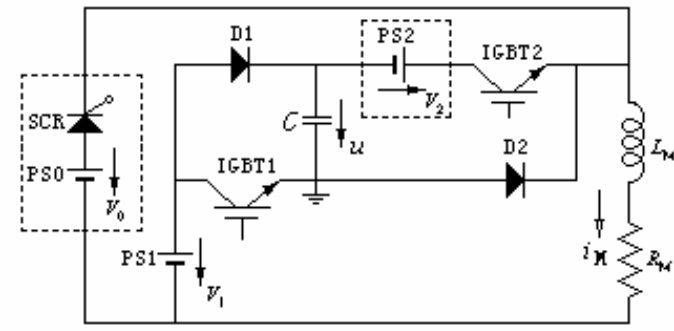

Figure 1: Fundamental Principle Diagram.

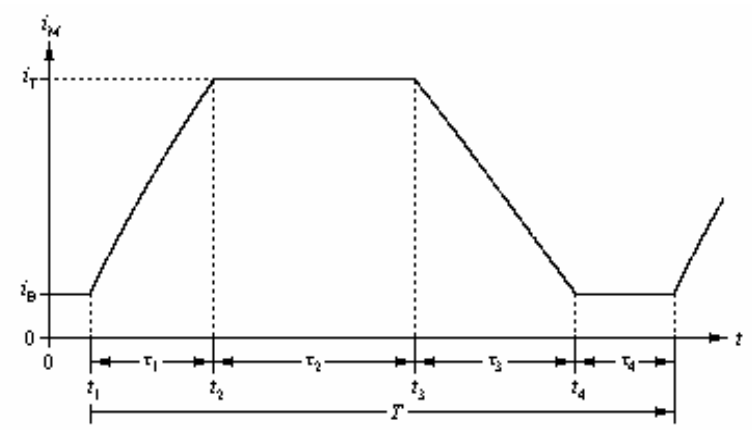

Figure 2: Load Current Wave Form.

Figure 2 is trapezoidal current waveform with nonzero flat bottom. Now we describe the principle and working process using this waveform as an example.

In Figure $1, L_{\mathrm{M}}$ and $R_{\mathrm{M}}$ are the inductance and resistance of the load magnet respectively. PS0, PS1, PS2 are DC regulators. $C$ is bank of capacitors for energy storage.

In a steady cycle, during the bottom period, the SCR has been on, and the voltage on the energy storage 
capacitor $C$ is charged to its full value of $\mathrm{u}=\mathrm{U}$. At $t=t_{1}$, IGBT1 and IGBT2 are turned on at the same time. The SCR turns off due to negative voltage across it. Capacitor $C$ discharges resonantly through $L_{\mathrm{M}}$, and the load current $i_{\mathrm{M}}$ rises. At $t=t_{2}, i_{\mathrm{M}}$ reaches the top current $i_{\mathrm{T}}$. IGBT1 turns off and D1 starts conducting. DC power supplies PS1 and PS2 maintain load current $i_{\mathrm{M}}=i_{\mathrm{T}}$ through D1 and IGBT2. At $t=t_{3}$, IGBT2 turns off. This shuts off the current path and generates a high voltage across $\mathrm{L}_{\mathrm{M}}$. Diode D2 starts conducting. Capacitor $C$ charges resonantly through $L_{\mathrm{M}}, \mathrm{PS} 1, \mathrm{D} 1$ and D2. Load current $i_{\mathrm{M}}$ starts to fall. The energy stored in the load inductance flows back to capacitor $C$. At $t=t_{4}, i_{\mathrm{M}}$ falls to $i_{\mathrm{B}}$. The SCR is turned on by an external signal, and the DC power supply PS0 maintains a flat load current $i_{\mathrm{M}}=i_{\mathrm{B}}$. D1 and D2 are off due to negative bias. This completes a full cycle, and the capacitor $C$ is charged to $U$ again.

When the system is turned on initially, the energy provided by the DC power supplies PS1 and PS2 exceeds that lost in the circuit. The voltage on capacitor $C$ and the load current build up over many cycles. After this transitional period the provided energy by the DC power supplies equals that lost in the circuit and the system enters a steady state.

In some cases, it is desirable to reduce the fall time. We can achieve this easily by reconfiguring the capacitor bank. For example, if we want to reduce it to one nth, $C$ is divided into $n$ units. Each unit has a capacitance of $C / n$. These units are connected with $3(n-1)$ diodes as shown in Figure 3. This ensures that all capacitors are discharged in parallel (in rising period) and charged in series (in falling period). The fall time will be reduced approximately to one $n$ th.

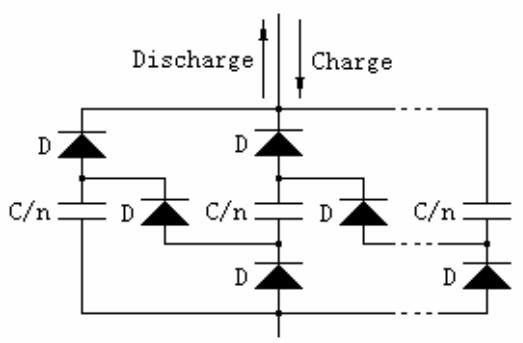

Figure 3: Diagram of discharge /charge of $n(C / n) \mathrm{s}$ in series/parallel.

\section{EXPERIMENTAL SET}

Two single module unit IGBTs (200A/1200V), a SCR rated at $50 \mathrm{~A} / 500 \mathrm{~V}$ and auxiliary circuits are installed in one IGBT/SCR driving-controller box. Other components in the main circuit are connected to the posts on the rear board.

Two 0 30V/0 30A DC voltage and current regulators are provided by the Magnet Field Measurement Group. One 0 12V/2A DC voltage and current regulator are provided by the Power Supply Group.
The electrolytic capacitors used as the energy storage capacitor $(10000 \mu \mathrm{F} / 100 \mathrm{~V}, 5$ in parallel) and two Diodes $(50 \mathrm{~A} / 700 \mathrm{~V})$ are spare parts borrowed from the Power Supply Group.

The load inductor consists of six filter inductors connected in series. It has the following parameters: $L_{\mathrm{M}}=82 \mathrm{mH}, R_{\mathrm{M}}=50 \mathrm{~m} \Omega$.

Construction of an experimental set started at the beginning of April last year. By the beginning of July we had completed several experiments and recorded the waveforms of load current and capacitor voltage (see Fig. 4 to Fig. 8). The basic principle of the suggested design is fully verified.

\section{EXPERIMENT RESULTS}

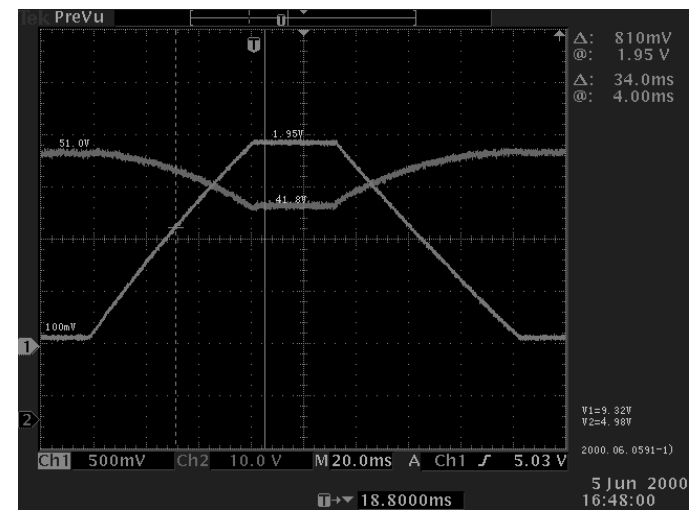

Figure 4: Trapezoidal waveform with $0.75 \mathrm{~V}$ flat bottom.

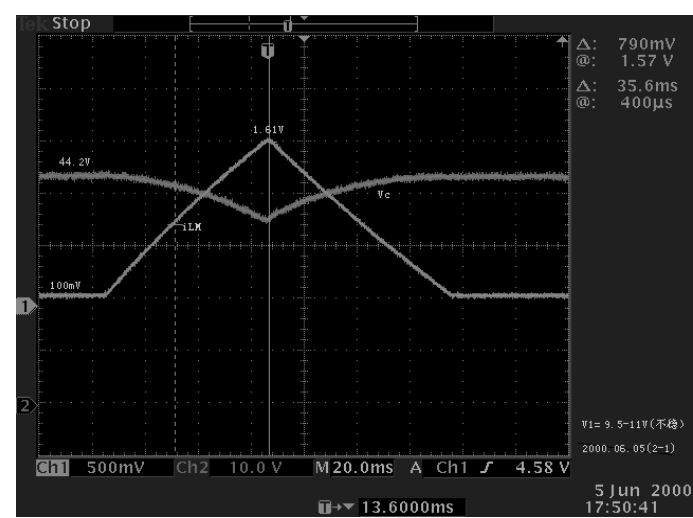

Figure 5: Triangular waveform with $0.75 \mathrm{~V}$ flat bottom.

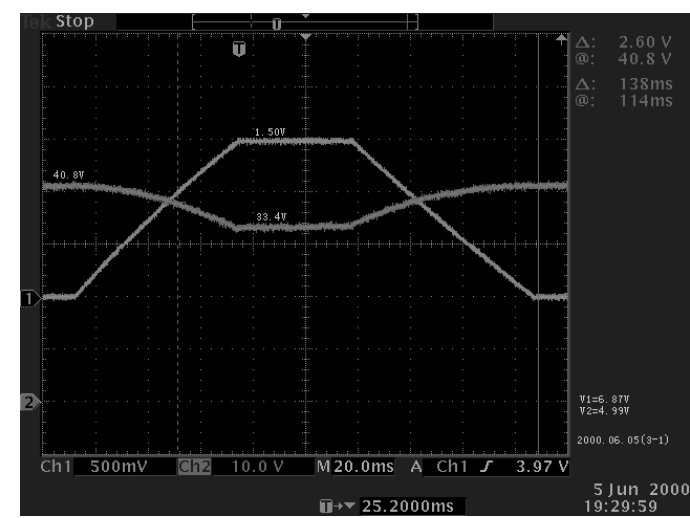

Figure 6: Trapezoidal waveform with 0V flat bottom. 


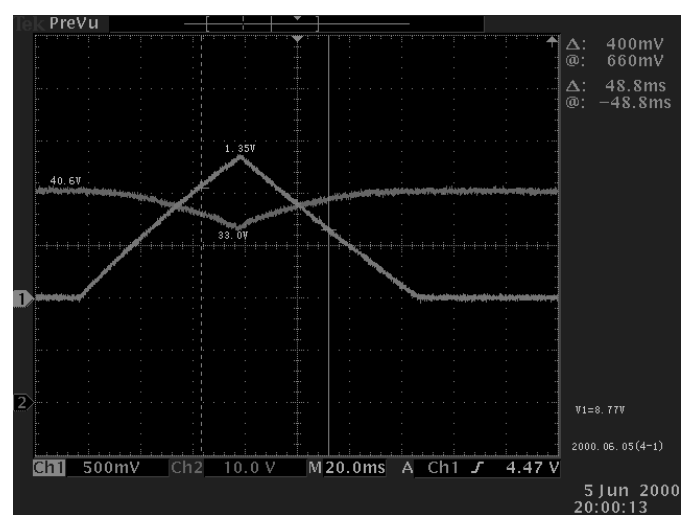

Figure 7: Triangular waveform with zero $0 \mathrm{~V}$ bottom.

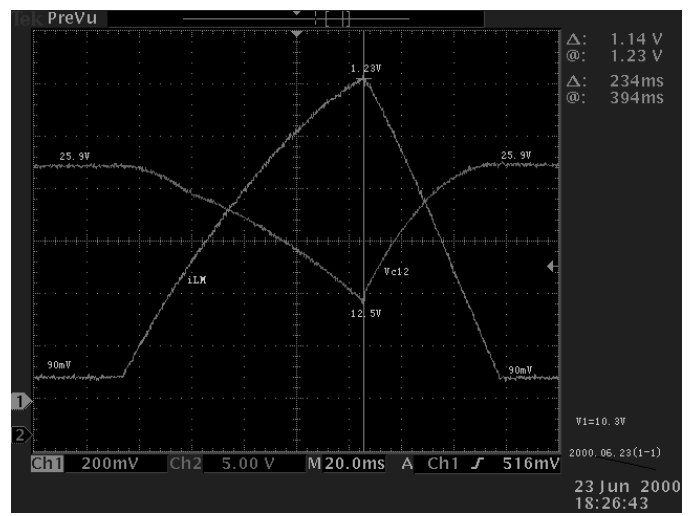

Figure 8: Triangular waveform with $0.75 \mathrm{~V}$ flat bottom. The fall time is reduced using two capacitors in series/parallel configuration.

\section{SUMMARY}

The flat bottom and top sections of the current waveform are directly provided by DC regulators. The rising and falling sections are generated by resonance of the energy storage capacitors, which are in series with DC regulators and the load inductance. Through properly set switch timings, transition between sections is optimized. The main characteristics are: energy store capacitor is charged automatically by the induced high voltage generated in the load inductor.

Using electrolytic capacitors as stored energy capacitors helps reduce cost and space. It is a viable alternative to other designs of slow cycling power supplies. It makes no strict demands on the capacitance value of the capacitor. So long as it is larger than a minimum value, the system can work properly. The larger the capacitance is, the lower the its charging voltage is, and the more linear the rising and falling sections of the load current are, and the lower the voltages across the IGBTs and diodes are, and also the lower the total power consumption is.

Utilizing IGBTs as the main switches, which can be turned off by external control, simplifies the design significantly. High power and current can be achieved by connecting IGBTs in series or parallel. The main technical challenge here is to balance the current and voltage distribution.

Using one DC regulator and one SCR providing flat bottom of the load current helps to eliminate a large and expensive choke, and accordingly makes the design more flexible and less expensive.

The waveform of the load current and cycle time can be easily adjusted by changing the output voltage of the DC regulators and the timing of the switches. If the desired waveform is triangular with zero flat bottom, only one DC regulator is required.

\section{REFERENCE}

[1] H.Sasaki, "Magnets for Fast-Cycling Synchrotrons", KEK Preprint 91-216, March 1992.

[2] A. Young and B. E. shafer, "Electrical Characteristics of the SSC Low-Energy Booster Magnet System", Conference Record IEEE, Particle Accelerator Conference, pp.1285-1287, 1993.

[3] C. Jack, etc. "Energy Storage Inductor for the Low-Energy Booster Resonant Power Supply System", Conference Record IEEE, Particle Accelerator Conference, pp.1297-1299, 1993.

[4] J. M. S. Kim and K. W. Reiniger, "Frequency-Domain Analysis of Resonant-Type Ring Magnet Power Supplies", Conference Record IEEE, Particle Accelerator Conference, pp.1112-1114, 1993.

[5] H. Sato, etc. "Upgrade of the Main Ring Magnet Power Supply for the KEK $12 \mathrm{GeV}$ Proton Synchrotron", Conference Record IEEE, Particle Accelerator Conference, pp.908-910, 1991.

[6] Masoud Fathizadeh, "Design and Simulation of High Accuracy Power Supplies for Injector Synchrotron Dipole Magnets", Conference Record IEEE, Particle Accelerator Conference, pp.917-919, 1991.

[7] Spring-8 Project. Part I, Facility Design 1990 [DRAFT], February 1991, pp.3-65 3-67.

[8] G. Karady, etc. "Resonant Power Supplies for a Large Synchrotron", IEEE Transactions on Nuclear Science, Vol. NS-32, pp.1480-1482, 1987.

[9] Ed Schneider "Test Results on Dual Resonant Power Supply with Flat Top and Flat Bottom Current", IEEE Transactions on Nuclear Science, Vol. NS-32, pp.1505-1507, 1987.

[10] W. F. Praeg, "Dual Resonant Power Supply with Flat-Bottom", IEEE Transactions on Nuclear Science, Vol. NS-30, No.4, pp.2873-2875, August 1983.

[11] M. R. Pavan Kumar \& J. M. S. Kim, "A Hybrid Multi-level Switching Converter for Ring-Magnet Power Supplies", IEEE Transactions on Nuclear Science, Vol. 43, No. 3, pp.1876-1883, June 1996.

[12] W. F. Praeg and D. McGhee, "Ring Magnet Power Supply for a $500 \mathrm{MeV}$ Synchrotron", Conference Record of the 1978 Industrial Applications Society, pp. 1118-1124. 